\title{
Feeding Intolerance in Preterm Neonates in Neonatal Intensive Care Unit, Padma General hospital, Dhaka
}

\author{
M A Islam ${ }^{1}$, S Begum², B H N Yasmeen ${ }^{3}$, J Ahmed ${ }^{4}$, M M Amin $^{5}$
}

\section{Abstract}

${ }^{1}$ Dr. Md. Atiqul Islam Registrar Dept. of pediatric Infectious Diseases \& Community Pediatrics Dhaka Shishu Hospital \& Visiting Consultant, NICU Padma General Hospital

2 Dr. Sheuly Begum Associate Professor Dept. of Gynae \& Obs. Enam Medical College, Savar

3 Prof. Dr. B H Nazma Yasmeen Professor \& Head Dept. of Pediatrics Northern International Medical College, Dhaka

${ }^{4}$ Dr. Jesmin Ahmed Co-ordinator Vaccination center Square Hospital Ltd.

${ }^{5}$ Dr. Md. Mahdi Amin Medical Officer NICU, Padma General Hospital

Correspondence

Dr. Md. Atiqul Islam

Registrar

Dept. of pediatric Infectious

Diseases \& Community Pediatrics Dhaka Shishu Hospital \& Visiting Consultant NICU, Padma General Hospital email: roman.atique@yahoo.com

Background: Premature infants frequently suffer from feeding intolerance and is highly associated to morbidity and mortality. It is a great challenge which have to face all the neonatologist. Feeding intolerance can be defined by difficulty in ingestion or digestion of the milk that causes a disruption in the enteral feeding plan due to the manifestation of some clinical symptoms. These symptoms include gastric residuals, emesis, abdominal distention, visible bowel loops, and change in the character of stool etc.

Objective : To determine the frequency of feeding intolerance in hospitalized preterm neonates.

Methods: This prospective study was carried out on preterm neonates admitted at neonatal intensive care unit of Padma General Hospital from 1st January 2016 to 31st December 2016. Inclusion criteria included Preterm infants of both genders from day 0 to day 28 of life and with gestational age (GA) of 28 to 36 weeks (estimated by 1 st day of maternal last menstrual period). Diagnosis of feeding intolerance depends on presence of one or more signs that leading to interruption of the enteral feeding regime of the preterm.

Results : The incidence of feeding intolerance in hospitalized preterm was $4.2 \%$

Conclusion: The percentage of feeding intolerance in our study is $4.2 \%$ of the preterm neonate.

Key words : Feeding intolerance, gastric residuals, necrotizing enterocolitis, incidence, preterm

Northern International Medical College Journal Vol. 8 No. 01 July 2016, Page 178-180

\section{Introduction}

Preterm infants have higher nutrient requirements than term infants because they have missed some or all of the third trimester of pregnancy which is a period of nutrient accretion and rapid growth. The fetus multiplies in weight five times from 24 weeks gestation to term (a period $<4$ months); in comparison term infants double their birth weight by 4-5 months. ${ }^{1}$

The American Academy of Pediatrics policy statement in 2012 on breastfeeding recommend human milk for term, preterm, and other highrisk infants either by direct breastfeeding and/or by expressed breast milk. ${ }^{2}$ Therefore establishing enteral feeding is a critical milestone during management of premature infant.

Preterm infants, who have feeding intolerance, get difficulty with the ingestion or digestion of formula or breast milk and that lead to disruption in the current enteral feeding plan. Symptoms of feeding intolerance include the presence of gastric residuals, vomiting, abdominal distention, visible bowel loops, diarrhea, or bloody stool. Apnea, bradycardia, and temperature instability are also included as symptoms of feeding intolerance. ${ }^{3}$ Feeding intolerance (FI) is often associated with nacrotizing enterocolitis, a leading cause of morbidity and mortality in preterm infant. ${ }^{4}$ Preterm infants who frequently suffer from feeding intolerance and subsequently exposed to parenteral nutrition for prolonged time. ${ }^{5}$

Factors that contribute to feeding intolerance include poor coordination of sucking and swallowing, incompetent lower esophageal sphincter, small gastric capacity, delayed gastric emptying time and intestinal hypo motility. ${ }^{6}$

An abnormal bacterial colonization may be a coexisting factor in feeding intolerance in newborn mainly due to dysfunction of the intestinal barrier, the immune responses, and sensory motor functions of the gut. Abnormal intestinal colonization, poor balance between microbiota,immune response and tolerance mechanisms may result in feeding intolerance in early postnatal life and in gastrointestinal disease in childhood. ${ }^{7}$ 
Considering all these factors we therefore try to find out how common is the feeding intolerance in preterm neonates in our context.

\section{Objective}

To determine the frequency of feeding intolerance in hospitalized preterm neonates.

\section{Materials and Methods}

This prospective study was carried out on 500 preterm neonates admitted to Neonatal Intensive Care Unit of Padma General Hospital, Dhaka from 1st January 2016 to 31st December 2016.

Inclusion criteria of the study were admitted preterm infants of both genders from day 0 to day 28 of life and with gestational age (GA) from 28 to 36 weeks (estimated by 1st day of maternal last menstrual period).

Diagnosis of feeding intolerance considered by the presence of one or more signs that leading to interruption of the enteral feeding regime of the preterm as increased gastric residuals $(>50 \%)$ of the previous feeding, greenish residual, hemorrhagic residual, emesis, abdominal distention (increase in abdominal girth by $2 \mathrm{~cm}$ or more in between feedings), bloody stool, diarrhea, visible bowel loops and apnea.

Exclusion criteria was neonates suffering from intestinal congenital anomalies, neonates with fulminating sepsis from onset, also neonates with milk allergy.

All the studied neonates were subjected to full history taking and full clinical examination.

\section{Statistical analysis}

The collected data were organized, tabulated, and statistically analyzed by using Statistical Package for Social Science (SPSS) version 16 .

\section{Ethical consideration}

Ethical approval for the study was obtained from the Ethical Committee for research, Padma General Hospital Limited,Dhaka and also written informed parental consent was obtained.

\section{Result}

Total 500 preterm neonates were enrolled in the study. Mean gestational age was found $32.1 \pm 2.3$ weeks with range from 28 to 36 weeks. The mean birth weight was found $1866.5 \pm 529.9$ gram with range from 950 to $2800 \mathrm{gram}$. The mean time of feeding intolerance diagnosis was found $6.1 \pm 4.2$ days with range from 2 to 15 days. Among them 16 (76.2\%) patients had feeding intolerance who was born in 28-32 weeks and 5 (23.8\%) was in 33-35 weeks of gestation. Only 21 (4.2\%) preterm had feeding intolerance. (Figure 1)

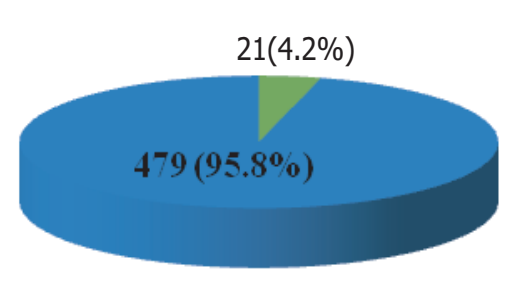

\begin{tabular}{|l|}
\hline Yes \\
No \\
\hline
\end{tabular}

Feeding intolerance

Figure 1: Feeding intolerance in preterm $(n=500)$

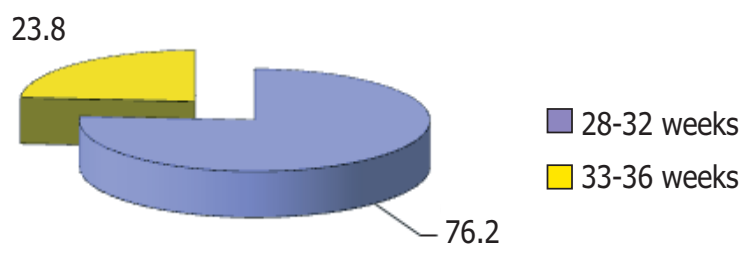

Feeding intolerance

Figure 2 : Feeding intolerance with gestational age $(n=21)$

Majority (95.2\%) patients had vomiting, 15(71.4\%) had abdominal distension, 5(23.8\%) had gastric residual, 4(19.0\%) had apnea, $4(19.0 \%)$ had greenish residual and $3(14.3 \%)$ had hemorrhagic residual.

Table 1 : Distribution of the study patients by signs of feeding intolerance $(n=21)$

$\begin{array}{lcc}\text { Signs of feeding intolerance } & \text { No. of patients } & \text { Percentage } \\ \text { Vomiting } & 20 & 95.2 \\ \text { Abdominal distension } & 15 & 71.4 \\ \text { Gastric residual } & 5 & 23.8 \\ \text { Apnea } & 4 & 19.0 \\ \text { Greenish residual } & 4 & 19.0 \\ \text { Hemorrhagic residual } & 3 & 14.3 \\ \text { * Multiple responses } & & \end{array}$

Table 2: Morbidities in the feeding intolerance cases $(n=21)$

$\begin{array}{lcc}\text { Morbidity } & \text { No. of patients } & \text { Percentage } \\ \text { Respiratory distress (RD) } & 12 & 57.1 \\ \text { Respiratory distress syndrome (RDS) } & 5 & 23.8 \\ \text { Necrotizing enterocolitis (NEC) } & 2 & 9.5 \\ \text { Transient tachypnea of the newborn (TTN) } & 1 & 4.8 \\ \text { Hypoxic ischemic encephalopathy (HIE) } & 1 & 4.8\end{array}$

* Multiple responses

In feeding intolerance $12(57.1 \%)$ patients was found with respiratory distress followed by $5(23.8 \%)$ with respiratory distress syndrome, 2(9.5\%) with necrotizing enterocolitis, $1(4.8 \%)$ with transient tachypnea of the newborn and 1 (4.8\%) with hypoxicischemic encephalopathy. 


\section{Discussion}

Feeding intolerance in the preterm infant is defined as follows: "Experiencing difficulty with the ingestion or digestion of formula or breast milk that causes a disruption in the current enteral feeding plan due to the manifestation of one or more defined clinical symptoms. ${ }^{3}$ These symptoms include gastric residuals, emesis, abdominal distention, visible bowel loops, and character of stool (diarrhea, guaiac positive or bloody). Apnea, bradycardia, and temperature instability are also included as symptoms of feeding intolerance but solely for the purposes of the nursing assessment in order to provide guidance on identification of potential progression to more serious complications such as pneumatosis intestinalis and necrotizing enterocolitis. ${ }^{3} \mathrm{FI}$ is one of the most common reasons to delay the advancement of enteral feeds or for suspension of feeds in preterm infants. $^{8}$

In this prospective study, we followed preterm infants who were admitted to Neonatal Intensive Care Unit of Padma General Hospital from 1st January 2016 to 31st December 2016 for the appearance of any signs of feeding intolerance. We assessed the incidence of feeding intolerance among this preterm neonates.

In this study, it was observed that mean gestational age was found $32.1 \pm 2.3$ weeks with range from 28 to 36 weeks. The mean birth weight was found 1866. $5 \pm 529.9 \mathrm{gram}(\mathrm{gm})$ with range from 950 to $2800 \mathrm{gram}$.

The mean time of feeding intolerance diagnosis was found $6.1 \pm 4.2$ days with range from 2 to 15 days. Study done by Zoppelli et al. showed that mean GA was 28.5 weeks and birth weight $1057 \mathrm{gm} .{ }^{9}$ In another study done by Albanna et al. showed that they had a mean GA of 32 weeks, a mean birth weight of $1500 \mathrm{gm}$ and a mean age at diagnosis of 9 days. ${ }^{10}$ The study of Carroll et al. ${ }^{11}$ had a mean GA of 30 weeks and mean age at diagnosis of 12 days. In the study of Yang et al. mean GA was found $26.6 \pm 2.1$ weeks and mean birth weight was $982.1 \pm 289.4 \mathrm{gm} .{ }^{12}$ Aydemir et al. found in their study that the mean GA was 29 weeks, mean birth weight of $950 \mathrm{gm}$ and a mean age of 14 days at diagnosis. ${ }^{13}$

In our study, from a total of 500 neonates, 21 preterm showed signs of feeding intolerance. The incidence of feeding intolerance in our study was $4.2 \%$. Majority $95.2 \%$ patients had vomiting, $71.4 \%$ had abdominal distension, $23.8 \%$ had gastric residual $>50 \%, 19.0 \%$ had greenish residual, $14.3 \%$ had hemorrhagic residual and $19.0 \%$ had apnea,. Sixteen (76.2\%) patients had feeding intolerance in 28-32 weeks and $5(23.8 \%)$ in 33-36 weeks of gestation.

In this study, among 21 feeding intolerance cases 12(57.1\%) patients was found morbidities with respiratory distress, $5(23.8 \%)$ with respiratory distress syndrome, $2(9.5 \%)$ with necrotizing enterocolitis, $1(4.8 \%)$ with transient tachypnea of the newborn and $1(4.8 \%)$ with hypoxic ischemic encephalopathy. One case died due to necrotizing enterocolitis.

Study done by Aydemir et al. showed that 3 patients had mild necrotizing enterocolitis (Bell's stage IB), 4 had definite necrotizing enterocolitis (Bell's stage IIB) and 3 had clinical signs consistent with advanced necrotizing enterocolitis (Bell's stage IIIA or IIIB). ${ }^{13}$

\section{Conclusion}

This prospective study included 500 preterm neonates found that the incidence of feeding intolerance was $4.2 \%$.

\section{References}

1. Fenton TR, Nasser R, Eliasziw M, Kim JH, Bilan D, Sauve R. Validating the weight gain of preterm infants between the reference growth curve of the fetus and the term infant. BMC Pediatr 2013;13:92.

2. American Academy of Pediatrics (AAP). Breastfeeding and the use of human milk. Policy statement. J Pediatr 2012;129:e827-41.

3. Moore TA, Wilson ME. Feeding intolerance: A concept analysis. Adv Neonatal Care 2011;11:149-54.

4. Thompson AM, Bizzarro MJ. Necrotizing enterocolitis in new born: Pathogenesis, prevention and management. Drugs. 2008;68(9): 12271238.

5. Ng PC, So KW, Fung KSC, Lee CH, Fok TF, Wong E. et al. Randomized control study of oral erythromycin for treatment of gastrointestinal dysmotility in preterm infant. Arch Dis Child Fetal Neonatal Ed 2001; 84:F177-F182.

6. Mansi Y, Abdelaziz N, Ezzeldin Z, Ibrahim R. Randomized controlled trial of a high dose of oral erythromycin for the treatment of feeding intolerance in preterm infants. Neonatology 2011;100:290-4.

7. Indrio F, Riezzo G, Cavallo L, Di Mauro A, Francavilla R. Physiological basis of food intolerance in VLBW. J Matern Fetal Neonatal Med 2011;24 Suppl $1: 64-6$.

8. Ramani $M$, Ambalavanan N. Feeding practices and necrotizing enterocolitis. Clin Perinatol 2013;40:1-10.

9. Zoppelli L, Güttel C, Bittrich HJ, Andrée C, Wirth S, Jenke A. Fecal calprotectin concentrations in premature infants have a lower limit and show postnatal and gestational age dependence. Neonatology 2012;102:68-74.

10. Albanna EA, Ahmed HS, Awad HA. Stool calprotectin in necrotizing enterocolitis. J Clin Neonatol 2014;3:16-9.

11. Carroll D, Corfield A, Spicer R, Cairns P. Faecal calprotectin concentrations and diagnosis of necrotising enterocolitis. Lancet 2003;361:310-1.

12. Yang $\mathrm{Q}$, Smith $\mathrm{PB}$, Goldberg RN, Cotten CM. Dynamic change of fecal calprotectin in very low birth weight infants during the first month of life. Neonatology 2008;94:267-71.

13. Aydemir G, Cekmez F, Tanju IA, Canpolat FE, Genc FA, Yildirim S, et al. Increased fecal calprotectin in preterm infants with necrotizing enterocolitis. Clin Lab 2012;58:841-4. 